\title{
Hypertension in Hemodialysis. An Overview on Physiopathology and Therapeutic Approach in Adults and Children
}

\author{
Rosario Cianci ${ }^{*}, 1$, Silvia Lai, Laura Fuiano ${ }^{2}$, Antonietta Gigante ${ }^{1}$, Paola Martina ${ }^{1}$, Biagio Barbano ${ }^{1}$, \\ Domenico Di Donato ${ }^{1}$, Gianfranco Clemenzia ${ }^{1}$, Pierangela Presta ${ }^{2}$, Paolo Gigliotti $^{2}$, \\ Michele Andreucci ${ }^{2}$, Alfredo Caglioti ${ }^{2}$ and Giorgio Fuiano ${ }^{2}$
}

\author{
${ }^{I}$ Viale dell 'Universita', 37, Dipartimento di Medicina Clinica, 6 Clinica Medica, 00100, Rome, Italy \\ ${ }^{2}$ University Magna Graecia, Catanzaro, Italy
}

\begin{abstract}
Chronic Kidney disease (CKD) patients, particularly those with end stage renal disease (ESRD), are at much higher risk of cardiovascular disease than the general population. Cardiovascular disease is by far the leading cause of morbidity and mortality in dyalisis patients, accounting for almost $40 \%$ of hospitalizations and almost $50 \%$ of deaths.

Hypertension is the single most important factor for the development of cardio and cerebrovascular complications. The etio-pathogenesis of hypertension in dialysis patients is multifactorial, sodium excess and extracellular volume expansion is the major factor in the development of hypertension, however there are other pathogenetic factors that should be considered, such as renin-angiotensin system hyperactivity, increased sympatic activity, altered endothelial cell function, hyperparathiroidism, and oxidative stress. The most important risk factors are anemia, hypoalbuminemia, chronic inflammation, prothrombotic factors, hyperomocisteinemia, vascular calcification and the traditional factors for cardiovascular risk (age, male gender, diabetes mellitus, dyslipidemia, smoke, physical inactivity, alcohol abuse).
\end{abstract}

Elevated blood pressure is frequent also in children on long term dialysis therapy. Data suggest that uremic factors or factors related to renal replacement therapy may be implicated in the pathogenesis of heart disease in adults and pediatric patients, because cardiovascular survival improves after transplantation.

The management of hypertension requires lifestyle modifications and control of volume status, with dietary salt and fluid restriction in combination with reduction of dialysate sodium concentration or with programmed "variable-sodium" dialysis.

The relationship between interdialytic weight and blood pressure is incompletely characterized; the dry weight (DW) method relies on the progressive reduction of the postdialysis body weight until blood pressure is normalized. There are several non clinical methods for evaluation DW such as measure the inferior vena cava diameter whit ultrasound, measure blood volume (BV) whit radiolabeled albumin and calculating post-hemodialysis BV from the change in hematocrit.

It may exist a lag time of several weeks between the normalization of the extracellular volume and blood pressure. It is related to the correction of the hemodinamic consequences of the extracellular volume overload.

The other problems to limit the possibility of correcting adequately volume expansion with dialysis are the use of antihypertensive drugs and the aggressive ultrafiltration required by short dialysis times. A possible treatment option for these patients may be increase time hemodialysis with slow, long hemodialysis; short, daily hemodialysis or nocturnal hemodialysis.

All classes of antihypertensive drugs can be used in dialysis patients, except the diuretics because inefficacy. Angiotensinconverting enzyme (ACE) and Angiotensin T1 receptor antagonist (AT1RA) appear to have the greatest ability to reduce left ventricular mass.

\section{INTRODUCTION}

Cardiovascular disease is the major cause of morbidity and mortality in patients with chronic kidney disease (CKD) Stage 5 and accounts for approximately $50 \%$ of deaths (KDOQI Guidelines) [1]. Overall, dialysis patients have a 10-20-fold increase in risk of death, compared with the general population [2]. In the recent HEMO study, the most com-

*Address correspondence to this author at the Viale dell 'Universita', 37, Dipartimento di Medicina Clinica, 6 Clinica Medica, 00100, Rome, Italy; Tel: +390649972074; E-mail: ciancirosario@tin.it mon cause of death in dialyzed patients was ischemic heart disease (20.4 per cent) followed by cardiac rhythm disorders (10.4 per cent), cerebrovascular disease ( 8.6 per cent), and infections ( 7.7 per cent) [1]. The cardiovascular risk factors among patients with CKD Stage 5 may be divided into those that are nonspecific to kidney disease but are more prevalent, and those that are specific to CKD Stage 5. The latter are undoubtedly important, since patients with CKD Stage 5 have disease-related risk factors such as anemia, hyperhomocysteinemia, hyperparathyroidism, oxidative stress, hypoalbuminemia, chronic inflammation, prothrombotic factors, among others. In addition, data suggest that uremic fac- 
tors, or factors related to renal replacement therapy (RRT)/dialysis may be implicated in the pathogenesis of heart disease in patients treated by dialysis, because cardiovascular survival improves after transplantation even in high-risk patients [3]. However, in patients on dialysis there is also increased prevalence of many traditional factors for cardiovascular risk (age, male gender, hypertension, diabetes, dyslipidemia, and physical inactivity); among these, hypertension is the most important risk factor for the development of cardio and cerebrovascular complications, in other words the leading cause of morbidity and mortality in dialysis patients. However, despite the universally recognized detrimental effect of hypertension in dialysis patients, 60-70 per cent of patients reported on clinical studies performed in Europe as well as in North America remains hypertensive while undergoing hemodialysis (HD) [4] or peritoneal dialysis (PD) treatment. This has been demonstrated in most survival statistics [5]. The main cause of such a poor control has been identified as the difficulty in obtaining optimal dry weight, coupled with large inter-dialytic weight gain and unrestricted, often excessive dietary sodium intake [6].

\section{DISCUSSION}

Despite the common opinion that blood pressure (BP) is an important predictor for cardiovascular (CV) morbidity and mortality in dialysis populations, several studies failed to identify hypertension as having a major influence on CV risk in large cohorts of ESRD patients [7]. Duranti et al. retrospectively studied 370 chronic uremic patients to determine the number of mortalities due to hypertension. A total of 168 patients were found to be normotensive and 202 as hypertensive. No significant difference in survival was found between normotensive and hypertensive patients, even when other prognostic variables were considered in the statistical analysis. By contrast, very few observational studies have associated hypertension with shorter survival and excellent BP control with increased survival. Hypertension treated with antihypertensive medications unequivocally reduces cerebrovascular risk, but demonstration of a survival benefit for cardiovascular mortality has proven more difficult to demonstrate. In addition, the question is further complicated by the "reverse-epidemiology" in dialysis patients, since recent observational studies have demonstrated a paradoxical relationship between increasing blood pressure and increasing mortality, showing that that a pre-dialysis systolic BP $<110 \mathrm{mmHg}$ is significantly associated with a relevant increase of mortality and that the lowest mortality rate can be registered in patients with a pre-dialysis systolic BP between 150 and $159 \mathrm{mmHg}$ [8, 9]. KalantarZadeh et al. have recently analyzed in depth this paradoxical phenomenon, studying a 15-month cohort of 40933 hemodialysis patients in the United States whose predialysis and postdialysis blood pressure values were recorded routinely during each hemodialysis treatment [10]. The hazard ratio for all causes death for a predialysis systolic $\mathrm{BP}<110 \mathrm{mmHg}$ was 1.60. Adjustment for the malnutrition-inflammation mitigated only partially this paradoxical association. Increased mortality in dialysis patients with low systolic BP were also reported by Stidley et al. in a cohort study of 16939 patients followed for 1 to 2 years [11], but an increased mortality after 3 years was observed in those with systolic BP greater than $150 \mathrm{mmHg}$. Therefore, at least three observations could be obtained from these studies as analyzed together: (a) the finding of a sponta- neously low BP in dialysis patients can be a negative prognostic marker, as a consequence of major $\mathrm{CV}$ disease or poor nutrition, the latter as a cause of what is known as the malnutrition-inflammation-atherosclerosis complex [10,12]; (b) consequently, patients with lower BP may represent a sicker population, because of significant systolic dysfunction and left ventricular dilatation and malnutrition [13]; (c) the short followup period can affect the results, because low pre-dialysis BP values are predictive of death only in association with short observation periods. In contrast, long-term observation showed that patients with normal BP have reduced mortality, compared with those with higher BP.

However, these data are capable of causing confusion and, therefore, it is not surprising that optimum blood pressure control has not been established. Different factors contribute to this uncertainty, the most important being (a) the concerns about the correct method of BP measurement in these patients; (b) the conflicting results of the most recent studies that do not allow to establish how low BP should be targeted.

The best method and timing of BP measurement in hemodialysis patients are subject to controversy. The lack of standardization of BP measurement in the dialysis unit may lead to misdiagnosis, so close attention must be paid to technical methods to obtain BP. Usually BP is measured just before and after the dialysis session. However, Coomer et al. observed that, although predialysis and postdialysis BP (recorded by dialysis nurses) correlated significantly with mean BP, predialysis systolic BP (SBP) overestimated mean SBP by an average of $10 \mathrm{~mm} \mathrm{Hg}$, while postdialysis SBP underestimated mean SBP by an average of $7 \mathrm{~mm} \mathrm{Hg}$ [14]. Thus, a composite of BP measurements over a period of 1 to 2 weeks rather than isolated readings should be used for guidance $[15,16]$. Interdialytic BP monitoring with an ambulatory BP monitor is the most reproducible method and is thought to best represent BP in dialysis patients. If available, ambulatory BP is a useful tool to evaluate the quality of BP control in the interdialytic period. Alternative forms of BP measurement, such as home BP, 20minute postdialysis BP, and short (3-hour to 4-hour) ambulatory blood pressure monitoring (ABPM), could prove useful when feasible or available [17]. However, continuous monitoring is warranted in patients suspected of poor control, such as those with large interdialytic weight gain [17], the results being reasonably reproducible. A further, additional problem in the evaluation of BP measurements is that uremic patients can lose the usual diurnal variation in blood pressure, possibly leading to nocturnal hypertension [18]. Thus, even patients supposed to be well controlled with daytime BP measurements may be at risk for hypertension-induced cardiovascular morbidity [19]. Due to the complexity of dialysis patients, the correct target of blood pressure goals cannot be exactly the same of that indicated for general population. It should ideally be individualized, taking into account the patient's cardiac and neurologic status, comorbidities and age. According to the clinical conditions of the patient a "normal" BP, defined as a mean ambulatory BP $<140 / 90 \mathrm{mmHg}$ during the day and $<120 / 80 \mathrm{mmHg}$ by night, is a reasonable target goal [20].

\section{PATHOGENESIS}

The etiology of hypertension in ESRD is multifactorial, as one or more of the following factors, summarized in Table $\mathbf{1}$, 
Table 1. The Etiopathogenesis of Hypertension in End Stage Renal Disease

\begin{tabular}{|c|c|c|}
\hline Etiology & Pathogenesis & Pathological Effects \\
\hline Hypervolemia & $\begin{array}{c}\text { Alteration sodium cellular transport } \\
\text { Excessive dietary sodium and fluid intake }\end{array}$ & $\begin{array}{l}\text { Sodium balance positive and extracellu- } \\
\text { lar volume expand }\end{array}$ \\
\hline Erythropoietin & Rapid correction whit severe anemia & Increased hematocrit \\
\hline Alterated endothelial cell function & $\begin{array}{c}\text { Elevated concentration of endothelin } 1 \\
\text { Accumulation inhibitors of the vascular endothelium-generated } \\
\text { vasoactive Nitric oxide deficiency }\end{array}$ & Increased systemic vascular resistance \\
\hline Hyperparathyroidism & $\begin{array}{l}\text { Increased intracellular calcium } \\
\text { Elevated calcium-phosphate ion product }\end{array}$ & Vascular calcification \\
\hline Calcification of the arterial tree & $\begin{array}{l}\text { Increased aortic stiffness } \\
\text { Reduced large and small vessel distensibility }\end{array}$ & $\begin{array}{l}\text { Increased systolic pressure and widening } \\
\text { of pulse pressure }\end{array}$ \\
\hline Increased sympathetic activity & $\begin{array}{l}\text { Accumulation of uremic metabolites stimulates the cardiovascular } \\
\text { centers in the brainstem }\end{array}$ & Increased systemic vascular resistance \\
\hline Renin-angiotensin system hyperactivity & Renal regional ischemia induced and inappropriate renin secretion & Increased systemic vascular resistance \\
\hline
\end{tabular}

play a role in individual patients. The balance of sodium, through its dietary intake and diminished renal excretion, becomes positive and the extracellular volume expands and the consequences are systemic hypertension and vascular and cardiac remodelling. A strong link between blood pressure variations and interdialytic body weight gain, show the important participation of volume state in modulating blood pressure in dialysis patients [21].

Hypervolemia. Volume expansion is perhaps the major factor in the development of hypertension in dialyzed patients. It leads to an elevation in BP via the combination of a rise in cardiac output and an inappropriately high systemic vascular resistance and/or from the secretion of ouabain-like inhibitors of Na-K-ATPase. The latter could lead to elevations in intracellular calcium, whose increase in vascular smooth muscle cells induce vasoconstriction. The importance of extracellular fluid expansion is witnessed by the observation that BP can be maintained within a normal range in the vast majority of dialysis patients by strict control of an extracellular volume excess (ECV) [18]. Correction of ECV overload has led to the dry weight (DW) concept, which is the post dialysis body-weight that allows BP to remain normal until the next dialysis session. In fact, regardless of the mechanism, removal of the excess sodium and attainment of DW can result in the normalization of BP in more than 60 percent of dialysis-dependent patients. However, this also means that a significant proportion of these patients remain hypertensive despite having reached their DW. Clinically it's very difficult to know if DW is achieved because this is a clinical definition. Therefore, besides volume expansion, other pathogenic factors should be considered, and, if possible, corrected.

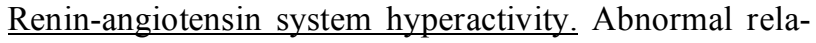
tionship between exchangeable sodium or BV and plasma renin activity (PRA) has been demonstrated in dialysis patients. This suggests that even "normal" plasma concentration of renin may, in some istance, be inappropriately high in the face of sodium excess or increased BV. The exact mechanism underlying such an inappropriate renin secretion is not completely understood, but it is probable that regional renal ischemia induced by scarring plays a major role.
Increased sympathetic activity. Sympathetic overactivity is a common finding in end-stage renal disease, correlating with the increase in both vascular resistance and systemic BP. This should originate in the diseased kidneys, since it is not seen in bilaterally nephrectomized patients. It has been hypothesized that the sympathetic hyperactivity is due to the accumulation of uremic metabolites that activate chemoreceptors within the kidney, eliciting a neural reflex that stimulates the cardiovascular centers in the brainstem. This hypothesis is supported by rat studies showing that renal deafferentation abrogates hypertension in the 5/6 nephrectomy model of chronic renal insufficiency. Additional potential mechanisms underlying Sympathetic overactivity includes increased levels of angiotensin II and asymmetrical dimethylarginine and contributes to hypertension and cardiovascular complications but its causes are not fully understood [22-24].

Altered endothelial cell function. An intriguing concept regarding the pathogenesis of hypertension in end-stage renal disease is the abnormal endothelial release of hemodynamically active compounds. The pathogenic role of increased concentrations of endothelin-1, a result of decreased renal clearance or endothelial cell damage has been proposed as a relevant hypertensive factor in these patients as well as the accumulation of endogenous inhibitors of the vascular endothelium-generated vasoactive compound nitric oxide such as asymmetric-dimethyl arginine (1-ADMA) that is an inhibitor of NO synthesis. This observation raises the possibility that NO deficiency may contribute to the development of hypertension in end-stage renal disease $[25,26]$.

Erythropoietin (EPO). An increase in BP of $10 \mathrm{mmHg}$ or more may occur in patients with renal failure who are treated with EPO. The risk is greatest in those with rapid correction of severe anemia and with preexistent hypertension. EPO can lead to an increase in blood pressure; the mechanisms of this effect are not entirely clear. By optimizing dialysis treatment, paying close attention to volume regulation, giving EPO subcutaneously and in a fashion to increase hematocrit gradually, the occurrence of BP increases can be minimized. Hypertension has not proved to be a serious general problem in the EPO treated patient [27]. 
Hyperparathyroidism. ESRD is frequently associated with secondary hyperparathyroidism which leads to increased intracellular calcium. It has been demonstrated that in patients with chronic renal failure is present a significant relation between intracellular calcium and PTH and between PTH and mean BP. In patients treated with vitamin D analogues mean BP decreased significantly and the changes in $\mathrm{BP}$ were strictly related with the changes in serum PTH and $\mathrm{Ca}^{++}$[28]. However, the results concerning the effects of parathyroidectomy on BP are conflicting [29,30], so that the role of hyperparathyroidism in the pathogenesis of hypertension in dialysis is still undefined. Extensive atherosclerosis and heavy vascular and valvular calcifications are common complications of ESRD and are very likely related to the high incidence of cardiovascular disease in these patients. The greatly increased incidence of cardiovascular disease is only partly explained by traditional risk factors for atherosclerosis. In ESRD, vascular calcification occurs both in the vascular intima layer and in the tunica media. Intimal calcification is disseminated and is characteristically associated with damaged and abnormally functioning endothelium, and macrophage and vascular smooth muscle cell (VSMC) infiltration typical of atherosclerosis. On the contrary, medial calcification occurs in patchy distribution and the most frequent cell types found in its vicinity are the VSMC and macrophage. The uremic state is associated with numerous metabolic abnormalities and endocrine disturbances primarily involving calcium and phosphorus metabolism. Furthermore, chronic kidney disease and dialysis are considered states of active and strong inflammatory response. These dysfunctions occur early in the course of renal failure and likely contribute to the development and progression of vascular calcification and atherosclerosis. For many years, vascular calcification was considered solely the result of a passive deposition of hydroxyapatite crystals in the arterial wall due to elevated calcium-phosphate ion product. However, a large body of evidence has now shown that this is a highly regulated process governed by factors that closely resemble calcium deposition in bone tissue. In fact, vascular calcification requires changes in the phenotype of VSMC and the expression of several proteins normally involved in bone metabolism. In the detection of vascular calcification in ESRD the modern imaging modalities and the therapeutic approaches are currently available to slow its progression.

Calcification of the arterial tree. Patients with end-stage renal disease are more likely to have an increase in pulse pressure and isolated systolic hypertension [15]. Why this occurs is incompletely understood but increased aortic stiffness appears to play an important role. Accelerated agerelated changes in vascular stiffness, together with factors peculiar to uremia, lead to loss of large and small vessel distensibility and profound changes in circulatory function that includes an increase in systolic pressure and widening of the pulse pressure. Pulse wave velocity is an integrated index of vascular function and structure, and is a major determinant of systolic hypertension, thereby increasing left ventricular afterload, left ventricular hypertrophy and left ventricular oxygen consumption. Decreased diastolic blood pressure, another consequence of arterial stiffening, is associated with decreased coronary perfusion contributing to ischaemic heart disease and evolution of adaptive into maladaptive left ventricular hypertrophy. Aortic stiffness measurements could serve as an important tool in identifying end-stage renal disease patients at higher risk of cardiovascular disease.

\section{HYPERTENSION IN PEDIATRIC PATIENTS}

Elevated BP is frequent also in children on long-term dialysis therapy. However, the prevalence of hypertension and status of BP control in these patients are lacking. Using the North American Pediatric Renal Transplantation Cooperative Study database, Mitsnefs et al determined the prevalence of hypertension and assessed risk factors for elevated BP during long-term dialysis therapy in children in a cohort including 3743 patients (age, 0 to 21 years). Uncontrolled hypertension was defined as BP equal to or greater than age, sex, and height-specific 95th percentiles; controlled hypertension was considered in children who were administered antihypertensive medications, but had BP less than the 95th percentile. A total of $76.6 \%$ of patients had either uncontrolled $(56.9 \%)$ or controlled $(19.7 \%)$ hypertension at baseline. Normotensive children at baseline had significant BP increases, whereas hypertensive children at baseline had significant BP decreases during the first year of dialysis therapy. BP did not change significantly after 1 year of dialysis therapy; $51 \%$ of patients had uncontrolled hypertension after 1 year of maintenance dialysis therapy. Logistic regression analysis shows that baseline hypertensive status and use of BP medications are both large significant risk factors for subsequent hypertension. Other risk factors include young age, acquired cause of renal failure, black race, duration of dialysis, and hemodialysis as a mode of renal replacement therapy [31]. Hypertension is very prevalent and difficult to control in children on dialysis therapy. In another study designed to identify risk factors for poor BP control in children on maintenance $\mathrm{HD}, 71$ subjects receiving HD in ten dialysis units participating in the Midwest of USA were evaluated. For each HD session, data on pre- and post treatment weights and BP were recorded. Hypertension, defined as mean BP $\geq 95$ th percentile, was found in 59 of the subjects. $15.5 \%$ had prehypertension, defined as mean BP between the 90th and 95th percentile. BP significantly decreased at the end of a dialysis session; however, only $35 \%$ of hypertensive subjects normalized their BP. Hypertensive subjects were younger than no-hypertensive, had higher serum phosphorus, and had more post-treatment weight above estimated dry weight perhaps reflecting their hypervolemic state. Logistic regression showed that younger age and higher serum phosphorus independently predicted hypertensive status. In conclusion, this study emphasizes the difficulty of BP control in pediatric HD patients [32]. However, also in a small cohort of pediatric patients on peritoneal dialysis the majority had BP abnormalities in which severity was most accurately assessed with ambulatory blood pressure monitoring. ABPM parameters were found to be significantly correlated with left ventricular mass index [33]. Therefore, hypertension in dialysis is a common and serious problem also in pediatric patients.

\section{MANAGEMENT}

The management of hypertension in the dialysis patients requires (a) lifestyle modifications and control of volume status with lifestyle modifications, (b) control of volume status with dialysis and (c) (if those measures are unsuccessful) the administration of antihypertensive drugs. 
Lifestyle modifications and control of volume status with lifestyle modifications. Lifestyle modification includes measures that help to reduce $\mathrm{BP}$ as well as other $\mathrm{CV}$ risk factors. These measures are similar to those traditionally prescribed to patients of general population, but are particularly important in high-risk patients as the dialysis patients are.

Weight reduction. Overweight (body mass index [BMI] $\left.=25-30 \mathrm{~kg} / \mathrm{m}^{2}\right)$ and obesity $\left(\mathrm{BMI}>30 \mathrm{~kg} / \mathrm{m}^{2}\right)$ have become mass phenomena with a pronounced upward trend in prevalence in most countries throughout the world and are associated with increased cardiovascular risk and poor survival. In patients with chronic kidney disease (CKD) undergoing maintenance hemodialysis an 'obesity paradox' has been consistently reported, i.e., a high BMI is incrementally associated with better survival. A similar obesity paradox has been described in patients with chronic heart failure as well as in 20 million members of other distinct medically 'at risk' populations in the USA. Possible causes of the reverse epidemiology of obesity include: (a) time-discrepancies between the competing risks for the adverse events that are associated with overnutrition and undernutrition; (b) sequestration of uremic toxins in adipose tissue; (c) selection of a gene pool favorable to longer survival in dialysis patients during the course of CKD progression, which eliminates over $95 \%$ of the CKD population before they commence maintenance dialysis therapy; (d) a more stable hemodynamic status; (e) alterations in circulating cytokines; (f) unique neurohormonal constellations; (g) endotoxin-lipoprotein interactions and (h) reverse causation [34]. This phenomenon, the obesity-survival paradox, is neither universally accepted nor completely understood. However, there is no doubt the a correct nutritional status with an appropriate BMI is associated whit a better outcome and help to keep BP controlled, because the "reverse" epidemiology of obesity in dialysis patients is mainly explained by mal-or undernutrition [35].

Salt and fluid. Dietary salt and fluid restriction is one of the most important measures to control BP in dialysis patients. The patients should adhere to a restricted salt diet (1000 to $1500 \mathrm{mg}$ of sodium/day), which also helps decrease thirst [36]. However, patient compliance is often suboptimal and a continuous education is essential to obtain a satisfactory adherence to a moderate dietary sodium (and water) intake.

Physical exercise. Regular exercise as a measure aimed at preventing renal failure results in a positive influence on blood pressure, a reduction in weight, reduction in peripheral insulin resistance, and a reduction in vessel rigidity. These effects result in improved cardiorespiratory function, muscular strength and quality of life [37].

Alcohol abuse. Alcoholism is not only common psychosocial disorder, but also an important CV risk factor. Moderation of alcoholic intake may also result bin remarakable $\mathrm{BP}$ reduction. Statistical modeling indicates that a $2 \%$ reduction in BP for all adults if alcohol intake is avoided. The impact of alcoholism on the care of patients undergoing hemodialysis has been addressed in USA using the Michigan Alcoholism Screening Test (MAST), a 25-item questionnaire that has been validated in multiple trials. Forty-five patients $(27.6 \%)$ scored 5 or greater on the MAST. The MASTpositive subjects were younger (age, $55+/-15$ years versus
$64+/-13$ years) and prevalently men (58\% versus $43 \%)$. A trend toward lower serum albumin level was evident in the men in the MAST-positive group. Therefore, such a high prevalence of alcoholism in the urban dialysis population suggests that dialysis patients should be screened for alcoholism and adequately treated $[38,39]$.

Tobacco use. Smoking has received surprisingly little research attention in dialysis populations, a group at monumental cardiovascular risk. In a recent study Medicare claims data were used to study associations between smoking and new-onset cardiovascular outcomes, and death in a prospective cohort $(\mathrm{N}=4024)$. Of the participants, $56.4 \%$ were lifetime nonsmokers, $3.6 \%$ were smokers with unknown current status, $20 \%$ had quit for more than 1 year, $5.8 \%$ had quit less than 1 year ago, and $14.2 \%$ were current smokers. Subjects with cardiovascular disease at baseline were more likely to be former smokers $(\mathrm{P}<0.001)$. When adjustment was made for baseline age, demographic variables, mode of dialysis therapy, and comorbidity, smoking status was associated with new-onset congestive heart failure (adjusted hazards ratio 1.59 comparing current to nonsmokers, $\mathrm{P}=0.004$ ), newonset peripheral vascular disease (adjusted hazards ratio $1.68, \mathrm{P}<0.001$ ), and mortality (adjusted hazards ratio 1.37 , $\mathrm{P}<0.001$ ). Former smokers, in contrast, had adjusted event risks similar to lifelong nonsmokers. The purpose of another study was to analyse both cross-sectional associations and how longitudinal changes in lifestyle factors and to estimate the hazard ratios for all-cause mortality for the changes in lifestyle factors and self-reported hypertension during the same period of time.

The authors found that continuing to smoke or being physically inactive or having hypertension at both points in time were all associated with higher hazard ratios for allcause mortality (1.6, 1.9 and 1.8 respectively). Therefore, smoking, altered fibrinogen levels and elevated systolic blood pressure, is a major, modifiable, cardiovascular risk factor in patients starting dialysis therapy $[2,40]$.

\section{TREATMENT}

Control of volume status. The total amount of sodium present in the body controls the extracellular volume. In advanced renal failure, sodium balance becomes positive and the extracellular volume expands. This leads to hypertension, and vascular changes that lead to adverse cardiovascular consequences in dialysis patients. Controlling the body sodium content and the extracellular volume allows one to better control hypertension and its consequences. This has led to the concept of dry weight, a crucial component of dialysis adequacy. It can be, in fact, defined DW as the postdialysis weight in which the patients remains normotensive without antihypertensive medication until the next dialysis [41], or as that body weiht at the end of dialysis below wich further reduction results in hypotension [35]. But the relative lack of accuracy of the clinical evaluation alone (and the physicians' progressive lack of interest in bedside examination) has led to several nonclinical methods of assessing DW in an effort to improve the assessment of fluid status in dialysis patients.

One problem is that volume expansion can persist even among those thought to have obtained dry weight. Katzarski et al., measuring the inferior vena cava diameter (IVCD) with ultrasound for the assessment of fluid status before, 
during and after the dialysis session as well as pre-HD blood volume (BV) with radiolabeled albumin, and calculating post-HD BV from the change in hematocrit found that (a) body weight, blood pressure, BV, and IVCD decreased in the entire population; (b) BV was significantly larger in the hypertensive patients than in the normotensive patients both before and after dialysis; (c) BV was correlated with the mean BP before and after HD; (d) IVCD was larger in the hypertensive than in the normotensive patients before and after HD; (e) BV and IVCD decreased in parallel during HD and increased during 2 hours after HD due to refilling of the intravascular space, indicating that changes in IVCD reflect changes in BV; (f) at the end of HD, several patients had IVCD below the reference range but IVCD increased during the following 1 to 2 hours, in some patients to values above the reference range. IVCD measured at the end or shortly after HD may therefore be misleading in assessing dry weight. However these are not useful measures in the clinical practice, because they require adequate re-equilibration after dialysis and prior to taking the measurement but these results confirm that extracellular fluid overload plays an important role in the pathogenesis of dialysis-associated hypertension [42]. The importance of inadequate correction of volume expansion as a cause of inadequate control of BP is also witnessed by the results of studies assessing extracellular volume by measuring pre and postdialysis plasma atrial natriuretic peptide (ANP). Predialysis ANP levels were higher in the hypertensive patients and postdialysis levels normalized only in those with dialysis-sensitive hypertension [43].

The other problems that limit the possibility of correcting adequately volume expansion with dialysis are the use of antihypertensive drugs and the aggressive ultrafiltration required by short dialysis times.

The use of antihypertensive drugs. In patients already on hypertensive agents the control of fluid status should be attained by gradually tapering the dosage of medications when BP progressively decreases as a result of ultrafiltration. If during the interdialytic period BP remain normal and it does not exceed 160/95 $\mathrm{mmHg}$ immediate before the next dialysis session, it is reasonable to keep antihypertensive therapy withheld [35].

The aggressive ultrafiltration. The discontinuous nature of hemodialysis causes saw-tooth volume fluctuations. The simplest way to improve the tolerance to rapid fluid removal during hemodialysis procedure is to perform prolonged and/or more frequent hemodialysis. Available therapeutic options to normalize blood pressure in these patients include dietary salt and fluid restriction in combination with reduction of dialysate sodium concentration. A possible treatment option for these patients may be long, slow hemodialysis ( 3 x $8 \mathrm{~h}$ per week); short daily hemodialysis (2-3 h, 7 times per week); nocturnal hemodialysis (6-7 times overnight per week). The results obtained with a standard regimen of eight hours regimen, three times a week in the dialysis center of Tassin, France, confirm this observation. In fact, in this center, almost all patients maintained normotension without medications. These results have to be attributed mainly to optimal volume control, although other factors, such as better control of uremia, decreased afferent nerve activity and efferent sympathetic activation $[44,45]$.
In addition, a better control of fluid and volume status with dialysis can be attained also reducing the dialysate sodium concentration. Flanigan et al. demonstrated that a "variable-sodium" dialysis program could reduce the blood pressure of dialysis patients without increasing dialysis morbidity. Dialysis with dialysate sodium of $140 \mathrm{mEq} / \mathrm{L}$ was compared with dialysis with a programmed exponential decrease of dialysate sodium from $155 \mathrm{mEq} / \mathrm{L}$ to $135 \mathrm{mEq} / \mathrm{L}$. Dialysate sodium was then held constant at $135 \mathrm{mEq} / \mathrm{L}$ for the final half hour of dialysis. Eighteen patients completed the 7-month study, each receiving 3.5 months of experimental and 3.5 months of standard therapy. Programmed "variable-sodium" dialysis resulted in a reduction in antihypertensive drug use without alterations in predialysis blood pressure, interdialytic weight gain, ultrafiltration tolerance, or the frequency of symptomatic dialysis cramps or hypotension $[46,47]$.

Therapy with antihypertensive drugs. A necessary premise to the use of drug therapy for hypertension in hemodialysis patients is this: even when the measures treated previously are unsuccessful (and frequently they are), dietary sodium restriction, lifestyle modifications and changes in the dialysis strategy, including a sensible dialysate sodium prescription, are indispensable in the success for an antihypertensive drug therapy program [48]. The current pattern and utilization of antihypertensive drugs was recently reported in the multicenter trial called HEMO study: in the United States $72 \%$ of the hemodyalisis patients received antihypertensive medication (48\% were prescribed calcium antagonists, $24 \%$ angiotensin-converting enzyme (ACE) inhibitors, and 21\% $\beta$-blockers) [49].

Choice of antihypertensive drugs. All classes of antihypertensive drugs can be used in dialysis patients, with the sole exception of diuretics, which are cot commonly used because of their lack of efficacy. Therefore, with the exceptions of diuretics, the criteria for drug selection are quite similar to those used in non-dialysis patients. Consequently, consideration should be given to the coexisting diseases, and, secondarily, to the patient's demographic characteristics, risk profile and lifestyle $[35,48]$. The presence of coexisting diseases should be carefully evaluated before choosing antihypertensive drugs. Table 2 summarizes some recommendations in the most common clinical situations.

ACE inhibitors. ACE inhibitors are usually well tolerated. They are particularly indicated in patients with heart failure due to systolic dysfunction and in many patients after an acute myocardial infarction. Their use has been associated with reduced mortality in hypertensive patients undergoing regular dialysis treatment. In a recent observational study, 60 patients had been treated with ACE inhibitors (treated group) and 66 patients had not (untreated group). Blood pressure reduction was not significantly different between the treated and untreated groups. Nevertheless, comparing the treated group with the untreated group, mortality was decreased significantly in the treated group, with a risk reduction of $52 \%$. In treated patients 65 years or younger, the absolute risk reduction of mortality was $79 \%$. Although further research is needed, these preliminary findings suggest that ACE inhibitors, independently of their antihypertensive effect, may dramatically reduce mortality among chronic hemodialysis patients 65 years or younger [50]. Their proba- 
Table 2. Choice of Antihypertensive Drugs in Consideration of Frequently Coexisting Diseases

\begin{tabular}{|c|c|c|}
\hline Clinical Situation & Preferred Drug & Not Recommended \\
\hline Angina Pectoris & $\begin{array}{c}\beta \text {-Blockers } \\
\text { Calcium antagonists } \\
\text { ACE inhibitors } \\
\text { AT1RA }\end{array}$ & Direct vasodilators \\
\hline Post-Myocardial Infarction & $\begin{array}{c}\text { Non intrinsic } \\
\text { sympathomimetic } \\
\text { activity- } \beta \text {-Blockers } \\
\text { ACE inhibitors } \\
\text { AT1RA }\end{array}$ & Direct vasodilators \\
\hline $\begin{array}{c}\text { Hypertrophic } \\
\text { cardiomyopathy with } \\
\text { diastolic dysfunction }\end{array}$ & $\begin{array}{l}\beta \text {-blockers } \\
\text { Diltiazem } \\
\text { Verapamil }\end{array}$ & $\begin{array}{l}\text { Direct vasodilators } \\
\alpha 1 \text {-blockers }\end{array}$ \\
\hline $\begin{array}{c}\text { Heart failure } \\
\text { (decreased left ventricular ejection fraction) }\end{array}$ & $\begin{array}{c}\text { AT1RA } \\
\text { ACE inhibitors } \\
\beta \text {-blockers }\end{array}$ & $\begin{array}{l}\text { Labetalol } \\
\text { Calcium-antagonists }\end{array}$ \\
\hline $\begin{array}{c}\text { Bradicardia } \\
\text { Heart block } \\
\text { Sick sinus syndrome }\end{array}$ & $\begin{array}{c}\text { Pacemaker } \\
\text { AT1RA } \\
\text { ACE inhibitors }\end{array}$ & $\begin{array}{c}\beta \text {-blockers; Labetalol } \\
\text { Verapamil } \\
\text { Diltiazem }\end{array}$ \\
\hline $\begin{array}{c}\text { Asthma } \\
\text { Chronic obstructive } \\
\text { pulmonary disease }\end{array}$ & $\begin{array}{c}\text { ACE inhibitors } \\
\text { AT1RA } \\
\text { Calcium antagonists }\end{array}$ & $\beta$-blockers; Labetalol \\
\hline
\end{tabular}

bly act by reducing mean arterial pressure, aortic pulse wave velocity, and aortic systolic pressure augmentation, as well as left ventricular hypertrophy $(\mathrm{LVH})$ and probably $\mathrm{C}$ reactive protein (CRP) and oxidant stress.

Safety. The most important side effects described with the use of ACE-inhibitors in end stage renal failure are hyperkalemia, anaphylactoid reactions with negatively charged hemodialysis membranes or other biomaterials and exacerbation of anemia. Use of ACE inhibitors in maintenance dialysis patients is associated with a significantly higher incidence of clinically important hyperkalemia by inhibition of residual excretion of potassium in the colon, or the cellular uptake of potassium [48]. This effect can be usually prevented with dietetic interventions, but weekly potassium control in the first month of dialysis and monthly measurements thereafter are recommended [51].

The report of anaphylactoid reactions is limited to some patient treated with ACE-inhibitors while undergoing dialysis with a high-flux AN69 dialyzer. This reaction was probably mediated by des-Arg9-braykinin and was triggered by negatively charged biomaterial (as AN69 is). Thus, it can be prevented just avoiding these biomaterial in patiens treated with ACE-inhibitors [52].

The use of ACE inhibitors may exacerbate anemia in patients with chronic renal disease by decreasing endogenous erythropoietin production and blunting the response to epoietin: however their effect on anemia in the dialysis patient is clinically little relevant [48].

Angiotensin-T1 receptors antagonists (AT1RA). These agents are perhaps more effective than ACE-inhibitors in causing regression of left ventricular hypertrophy in patients with end stage renal disease, but the current experience is limited. However, it is unquestionable that both ACE inhibitors and AT1RA are underused, as demonstrated by a recent retrospective analysis of the Minnesota Heart Survey but it is probable that a more extensive use of these drugs could be reduce cardiovascular events and mortality in patients on chronic maintenance hemodialysis and therefore improves the prognosis of these [53].

\section{CONCLUSION}

The etio-pathogenesis of hypertension in dialysis patients is multifactorial, sodium excess and extracellular volume expansion is the major factor in the development of hypertension, however there are other pathogenetic factors should be considered, such as renin-angiotensin system hyperactivity, increased sympatic activity, altered endothelial cell function, hyperparathiroidism, and oxidative stress. The most important risk factors are anemia, hypoalbuminemia, chronic inflammation, prothrombotic factors, hyperomocisteinemia, vascular calcification and the traditional factors for cardiovascular risk (age, male gender, diabetes mellitus, dyslipidemia, smoke, physical inactivity, alcohol abuse). The management of hypertension requires lifestyle modifications (regular physical exercise, alcohol and tobacco avoidance), a good nutrition with an appropriated BMI and control of volume status, whit dietary salt and fluid restriction. Blood pressure is the key indicator of ECV and is used to set the body weight target, usually with a lag time. The problems that limit the possibility of correcting adequately volume expansion with dialysis are the use of antihypertensive drugs and the aggressive ultrafiltration required by short dialysis times. A possible treatment option for these patients may be 
increase time hemodialysis with slow, long hemodialysis; short, daily hemodialysis or nocturnal hemodialysis and gradually tapering the dosage of medications when BP progressively decreases as a result of ultrafiltration. All classes of antihypertensive drugs can be used in dialysis patients, except the diuretics because inefficacy. ACE inhibitors and AT1RA appear to have the greatest ability to reduce left ventricular mass.

\section{ABBREVIATIONS}

\begin{tabular}{|c|c|c|}
\hline CKD & $=$ & Chronic Kidney disease \\
\hline ESRD & $=$ & End stage renal disease \\
\hline DW & $=$ & Dry weight \\
\hline BV & $=$ & Blood volume \\
\hline $\mathrm{ACE}$ & $=$ & Angiotensin-converting enzyme \\
\hline AT1RA & $=$ & Angiotensin T1 receptor antagonist \\
\hline RRT & $=$ & Renal replacement therapy \\
\hline HD & $=$ & Hemodialysis \\
\hline PD & $=$ & Peritoneal dialysis \\
\hline $\mathrm{BP}$ & $=$ & Blood pressure \\
\hline $\mathrm{CV}$ & $=$ & Cardiovascular \\
\hline SBP & $=$ & Systolic BP \\
\hline ABPM & $=$ & Ambulatory blood pressure monitoring \\
\hline $\mathrm{ECV}$ & $=$ & Extracellular volume excess \\
\hline PRA & $=$ & Plasma renin activity \\
\hline 1-ADMA & $=$ & Asymmetric-dimethyl arginine \\
\hline EPO & $=$ & Erythropoietin \\
\hline VSMC & $=$ & Vascular smooth muscle cell \\
\hline BMI & $=$ & Body mass index \\
\hline MAST & $=$ & Michigan Alcoholism Screening Test \\
\hline IVCD & $=$ & Inferior vena cava diameter \\
\hline ANP & $=$ & Atrial natriuretic peptide \\
\hline $\mathrm{ACE}$ & $=$ & Angiotensin-converting enzyme \\
\hline LVH & $=$ & Left ventricular hypertrophy \\
\hline CRP & $=$ & C-reactive protein \\
\hline
\end{tabular}

\section{REFERENCES}

[1] Rocco MV, Yan G, Gassman J, et al. Comparison of causes of death using HEMO Study and HCFA end-stage renal disease death notification classification systems. The National Institutes of Health-funded Hemodialysis. Health Care Financing Administration. Am J Kidney Dis 2002; 39: 146-53.

[2] Foley RN, Herzog CA, Collins AJ. Smoking and cardiovascular outcomes in dialysis patients: the United States Renal Data System Wave 2 study. Kidney Int 2003; 63: 1462-7.

[3] K/DOQI clinical practice guidelines for cardiovascular disease in dialysis patients. Am J Kidney Dis 2005; 45: S1-153.

[4] Grekas D, Kalevrosoglou I, Karamouzis M, et al. Effect of sympathetic and plasma renin activity on hemodialysis hypertension. Clin Nephrol 2001; 55: 115-20.

[5] Light P. Current understanding of optimal blood pressure goals in dialysis patients. Curr Hypertens Rep 2006; 8: 413-9.

[6] Locatelli F, Covic A, Chazot C, et al. Hypertension and cardiovascular risk assessment in dialysis patients. Nephrol Dial Transplant 2004; 19: 1058-68.
[7] Duranti E, Imperiali P, Sasdelli M. Is hypertension a mortality risk factor in dialysis? Kidney Int Suppl 1996; 55: S173-74.

[8] Port FK, Hulbert-Shearon TE, Wolfe RA, et al. Predialysis blood pressure and mortality risk in a national sample of maintenance hemodialysis patients. Am J Kidney Dis 1999; 33: 507-17.

[9] Zager PG, Nikolic J, Brown RH, et al. "U" curve association of blood pressure and mortality in hemodialysis patients. Kidney Int 1998; 54: 561-9.

[10] Kalantar-Zadeh K, Kilpatrick RD, McAllister CJ, et al. Reverse epidemiology of hypertension and cardiovascular death in the hemodialysis population: the 58th annual fall conference and scientific sessions. Hypertension 2005; 45: 811-7.

[11] Stidley CA, Hunt WC, Tentori F, et al. Changing relationship of blood pressure with mortality over time among hemodialysis patients. J Am Soc Nephrol 2006; 17: 513-20.

[12] Nurmohamed SA, Nube MJ. Reverse epidemiology: paradoxical observations in haemodialysis patients. Neth J Med 2005; 63: 37681.

[13] Agarwal R. Hypertension in chronic kidney disease and dialysis: pathophysiology and management. Cardiol Clin 2005; 23: 237-48.

[14] Coomer RW, Schulman G, Breyer JA, et al. Ambulatory blood pressure monitoring in dialysis patients and estimation of mean interdialytic blood pressure. Am J Kidney Dis 1997; 29: 678-84.

[15] Tripepi G, Fagugli RM, Dattolo P, et al. Prognostic value of 24hour ambulatory blood pressure monitoring and of night/day ratio in nondiabetic, cardiovascular events-free hemodialysis patients. Kidney Int 2005; 68: 1294-302.

[16] Zoccali C, Tripepi G. Ambulatory monitoring and clinic BP measurements for predicting left ventricular mass in patients with chronic renal diseases. Nephrol Dial Transplant 1999; 14: 240-1.

[17] Sankaranarayanan N, Santos SF, Peixoto AJ. Blood pressure measurement in dialysis patients. Adv Chronic Kidney Dis 2004; 11: $134-42$.

[18] Santos SF, Peixoto AJ. Hypertension in dialysis. Curr Opin Nephrol Hypertens 2005; 14: 111-8.

[19] Liu M, Takahashi H, Morita Y, et al. Non-dipping is a potent predictor of cardiovascular mortality and is associated with autonomic dysfunction in haemodialysis patients. Nephrol Dial Transplant 2003; 18: 563-9.

[20] Henrich DL, Mailloux LU. Hypertension in dialysis. UpToDate, Rose, BD (Ed), UpToDate, Waltham, MA, 2006.

[21] Kooman JP, van der Sande FM, Leunissen KM. Role of sodium and volume in the pathogenesis of hypertension in dialysis patients. Reflections on pathophysiological mechanisms. Blood Purif 2004; 22: 55-9.

[22] Yoshino M, Kuhlmann MK, Kotanko P, et al. International differences in dialysis mortality reflect background general population atherosclerotic cardiovascular mortality. J Am Soc Nephrol 2006; 17: 3510-19.

[23] Lozano A, Benavides B, Quiros P, et al. Control of arterial hypertension by means of a regimen of hemodialysis on alternate days (HDAA or EODD: "Every Other Day Dialysis") versus 2 conventional regiments of 4 and 5 hours per session 3 times a week with 72 hours without sessions during the weekends. Nefrologia 2006; 26: 695-702.

[24] Augustyniak RA, Tuncel M, Zhang W, et al. Sympathetic overactivity as a cause of hypertension in chronic renal failure. J Hypertens 2002; 20(1): 3-9.

[25] Zoccali C. Endothelial damage, asymmetric dimethylarginine and cardiovascular risk in end-stage renal disease. Blood Purif 2002; 20: 469-72.

[26] Zoccali C, Mallamaci F, Tripepi G. Traditional and emerging cardiovascular risk factors in end-stage renal disease. Kidney Int Suppl 2003; 85: 105-10.

[27] Hamburger RJ, Christ PG, Morris PA, et al. Hypertension in dialysis patients: does CAPD provide an advantage? Adv Perit Dial 1989; 5: 91-6.

[28] Raine AE. Can cardiovascular complications be prevented in dialysis patients? Adv Nephrol Necker Hosp 1996; 25: 317-39.

[29] Ifudu O, Matthew JJ, Macey LJ, et al. Parathyroidectomy does not correct hypertension in patients on maintenance hemodialysis. Am J Nephrol 1998; 18: 28-34.

[30] Coen G, Calabria S, Bellinghieri G, et al. Parathyroidectomy in chronic renal failure: short- and long-term results on parathyroid function, blood pressure and anemia. Nephron 2001; 88: 149-55. 
[31] Mitsnefes M, Stablein D. Hypertension in pediatric patients on long-term dialysis: a report of the North American Pediatric Renal Transplant Cooperative Study (NAPRTCS). Am J Kidney Dis 2005; 45: 309-15.

[32] VanDeVoorde RG, Barletta GM, Chand DH, et al. Blood pressure control in pediatric hemodialysis: the Midwest Pediatric Nephrology Consortium Study. Pediatr Nephrol 2007; 22: 547-53.

[33] Ozcakar ZB, Yalcinkaya F, Tutar E, et al. Hypertension and left ventricular hypertrophy in pediatric peritoneal dialysis patients: ambulatory blood pressure monitoring and echocardiographic evaluation. Nephron Clin Pract 2006; 104: c101-6.

[34] Kalantar-Zadeh K, Kopple JD. Obesity paradox in patients on maintenance dialysis. Contrib Nephrol 2006; 151: 57-69.

[35] Campese VM TA. Hypertension in dialysis patients. Philadelphia, Lippincott Williams \& Wilkins, 2004.

[36] Ahmad S. Dietary sodium restriction for hypertension in dialysis patients. Semin Dial 2004; 17: 284-7.

[37] Kosmadakis GC, Zerefos N. Physical exercise in dialysis patients. Int J Artif Organs 2007; 30: 429-34.

[38] Hegde A, Veis JH, Seidman A, et al. High prevalence of alcoholism in dialysis patients. Am J Kidney Dis 2000; 35: 1039-43.

[39] Gueye AS, Chelamcharla M, Baird BC, et al. The association between recipient alcohol dependency and long-term graft and recipient survival. Nephrol Dial Transplant 2007; 22: 891-8.

[40] Johansson SE, Sundquist J. Change in lifestyle factors and their influence on health status and all-cause mortality. Int J Epidemiol 1999; 28(6): 1073-80.

[41] Charra B, Laurent G, Chazot C, et al. Clinical assessment of dry weight. Nephrol Dial Transplant 1996; 11(Suppl 2): 16-19.

[42] Katzarski KS, Nisell J, Randmaa I, et al. A critical evaluation of ultrasound measurement of inferior vena cava diameter in assessing dry weight in normotensive and hypertensive hemodialysis patients. Am J Kidney Dis 1997; 30: 459-65.

[43] Fishbane S, Natke E, Maesaka JK. Role of volume overload in dialysis-refractory hypertension. Am J Kidney Dis 1996; 28: $257-$ 61.

[44] Charra B, Terrat JC, Vanel T, et al. Long thrice weekly hemodialysis: the Tassin experience. Int J Artif Organs 2004; 27: 265-83.

[45] Henrich WL, Mailloux LU. Hypertension in dialysis patients. Waltham, MA, UpToDate, 2007.

[46] Flanigan MJ, Khairullah QT, Lim VS. Dialysate sodium delivery can alter chronic blood pressure management. Am J Kidney Dis 1997; 29: 383-91.

[47] Flanigan M. Dialysate composition and hemodialysis hypertension. Semin Dial 2004; 17: 279-83.

[48] Horl MP, Horl WH. Drug therapy for hypertension in hemodialysis patients. Semin Dial 2004; 17: 288-94.

[49] Leypoldt JK, Cheung AK, Delmez JA, et al. Relationship between volume status and blood pressure during chronic hemodialysis. Kidney Int 2002; 61: 266-75.

[50] Efrati S, Zaidenstein R, Dishy V, et al. ACE inhibitors and survival of hemodialysis patients. Am J Kidney Dis 2002; 40: 1023-9.

[51] Knoll GA, Sahgal A, Nair RC, et al. Renin-angiotensin system blockade and the risk of hyperkalemia in chronic hemodialysis patients. Am J Med 2002; 112: 110-14.

[52] Amore A, Guarnieri G, Atti M, et al. Use of alkaline rinsing solution to prevent hypersensitivity reactions during hemodialysis: data from a multicentre retrospective analysis. J Nephrol 1999; 2: 383-9.

[53] Berger AK, Duval S, Manske C, et al. Angiotensin-converting enzyme inhibitors and angiotensin receptor blockers in patients with congestive heart failure and chronic kidney disease. Am Heart J 2007; 153: 1064-73.

(C) Cianci et al.; Licensee Bentham Open.

This is an open access article licensed under the terms of the Creative Commons Attribution Non-Commercial License (http://creativecommons.org/licenses/by$\mathrm{nc} / 3.0 /$ ) which permits unrestricted, non-commercial use, distribution and reproduction in any medium, provided the work is properly cited. 